\title{
Hepatic amyloidosis - primary AL type, sub nephrotic proteinuria and budd chairi syndrome - A nugget
}

\author{
Arun Kumar Narayanan', Satish Balan², Sandeep Patil', Praveen Murlidharan², \\ Ajith Krishnan Nair ${ }^{3}$, Shabeerali Thudikkodan Usman ${ }^{4}$, Manoj Krishnan Sarojam ${ }^{5}$, \\ Venupgopal Bhaskaran Pillai ${ }^{4}$, Ramdas Pisharody ${ }^{2}$
}

${ }^{1}$ Resident, Department of Nephrology, Consultant, ${ }^{2}$ Departments of Nephrology, ${ }^{3}$ Medical Gastroenterology, ${ }^{4}$ Surgical Gastroenterology, and ${ }^{5}$ Radiology, Kerala Institute of Medical Sciences, Thiruvananthapuram, Kerala, India

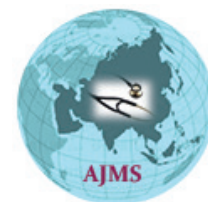

A B S T R A C T

We describe a case of 62-year-old gentleman presenting with abdominal pain associated with loss of weight nocturia, edema of feet, constipation, altered sleep rhythm and dyspepsia. On evaluation he had hepatomegaly with raised alkaline phosphatase and raised GGT levels with normal transaminases and bilirubin. On imaging he had diffuse enlargement of liver with heterogeneous contrast uptake in liver. His viral marker and autoimmune markers were negative. Liver biopsy depicted deposition of amorphous eosinophilic substance within the sinusoids which revealed apple green birefringence on polarizing microscopy after Congo red staining; Congophilia persisted even after treating with $\mathrm{KMnO}_{4}$. Abdominal fat pad was negative for amyloid deposit. Cardiac evaluation was unremarkable and renal evaluation showed Subnephrotic proteinuria and microhematuria. Serum and urine immunofixation electrophoresis showed positive kappa, lambda and gamma globulin. Immunoperoxidase staining for serum amyloid associated protein for secondary amyloidosis was negative from liver biopsy. Here we report a case of primary hepatic amyloidosis that presented with features of hepatic vein obstruction. The association of Budd-Chiari's syndrome, with amyloidosis may be related to the increased risk of thrombosis observed in the latter disease also due to loss of anticoagulants due to significant proteinuria. Patient could not be treated either with chemotherapy or with surgery. We lost him for progressive liver failure.

Key words: Amyloidosis, Fbrillary protein, Progressive liver failure

\section{Access this article online}

Website:

http://nepjol.info/index.php/AJMS DOI: $10.3126 /$ ajms.v8i2.16332 E-ISSN: 2091-0576 P-ISSN: 2467-9100

\section{INTRODUCTION}

Amyloidosis is a disease characterized by the deposition of altered proteins in tissues. ${ }^{1}$ It is a pathological process encompassing a spectrum of disease that results from the extracellular deposition of fibrillar amyloid protein. ${ }^{2}$ It is classified as primary or secondary disease. In both primary and secondary amyloidosis, the most commonly involved organ system is the gastrointestinal system, with the colon being the most frequently involved organ. ${ }^{3}$ Hepatic involvement, secondary amyloidosis is common diffuse infiltration is the rule, but primary systemic amyloidosis (amyloid light [AL] chain) is an uncommon entity. ${ }^{1}$ It may result in massive hepatomegaly, elevated serum alkaline phosphatase (ALP) levels and rapidly progressive liver failure. $^{4}$

To our knowledge, cases of amyloidosis involving the liver being presented as hepatic vein thrombosis have not been described previously but with IVC thrombus has been reported but that is in hepatic and GI systemic amyloidosis but not primary amyloidosis. ${ }^{5,6}$ Here we report a case of primary hepatic amyloidosis (HA) that presented with features of hepatic vein obstruction. The association of Budd-Chiari's syndrome with amyloidosis may be related to the increased risk of thrombosis observed in the latter disease. 


\section{THE CASE}

A Sixty-two years old gentleman, a landlord, presented with significant smoking and ethanol intake, with increased frequency of micturition, nocturia, significant weight loss $10 \mathrm{kgs}$ in a span of 2 months, constipation, altered sleep rhythm, Past h/o umbilical hernia surgery, with ascites and significant weight loss.

In 2015 He was operated for umbilical hernia, h/o spasmodic abdominal pain-2 months, diffuse, pain lateralized to left side of the abdomen. No other comorbidities.

Asthenic built, with loss of buccal pad of fat and undernourished BMI- $24.56 \mathrm{Kg} / \mathrm{m}^{2}$. He had Pallor, Mild icterus, Clubbing-B/L painless, carpal clubbing grade II, pedal edema grade II. Beefy red tongue. Sparse \& dry hair, sunken eyes, poor oral hygiene, JVP elevated-prominent " $\mathrm{a}$ " waves, hepatojugular reflux + . No signs of hepatocellular failure. Veins engorged, non tortuous filling away from the umbilicus.

On examination of per abdomen is was uniformly distended, flanks full, A transverse clean, hyperpigmented scar, measuring $15-20 \mathrm{~cm}$ across the umbilicus seen in the hypogastric region. A firm- hard mass, coarse, sharp, non tender, non pulsatile felt $5 \mathrm{~cm}$ in the midaxillary line from below sub costal margin, $8 \mathrm{~cm}$ in the midclavicular line below the sub costal margin and $5 \mathrm{~cm}$ in the mid sternal line from Xiphisternum and $3 \mathrm{~cm}$ in the left midclavicular line below sub costal margin.

Investigations revealed Total Bil -1.9, Indirect-1.1, AST-90, ALT-41, AST/ALT=90/41, ALP-560, alb-2.3, TP-5.3, Glb-3, GGT- 553. SAAG-2.2 -transudate, ANA-positive 1:100 dilution pattern - nucleoplasm, chromosome. AFP- 1.85, CA-19-9-46.68 (Raised), CEA-4.23-Normal, Ceruloplasmin- 50.9. CRP-14, ESR-15, Ferritin- 477 (increased), TSAT- 31.5\%, INR- 1.88, PTT-41.2, PT- 23.8. ECG - WNL. Ascitic fluid analysis, alb-0.10, LDH -38, Pr-0.4, cytology no malignant cells. Microscopy- scattered mesothelial and macrophages and lymphocytes. Culture sterile, ADA - negative.

Upper GI Endoscopy- 2 X 3 column, oesophageal varies, gastropathy, duodenopathy. Transjugular liver Biopsy and HVPG done. HVPG -normal, TEG- normal thrombus formation. Liver biopsy showed deposition of amorphous eosinophilic material in the sinusoids causing atrophy of liver plates, portal tracts show similar deposits no fibrosis or inflammation seen, no lobular inflammation Figure 1a Material was weakly PAS positive Figure $1 \mathrm{~b}$. The material was stained with congo red, Apple green birefringence was seen with polarizer Figure 1c. It persisted to have Congophilia even after treating with $\mathrm{KMnO} 4$ Figure 1d. PASD stain is

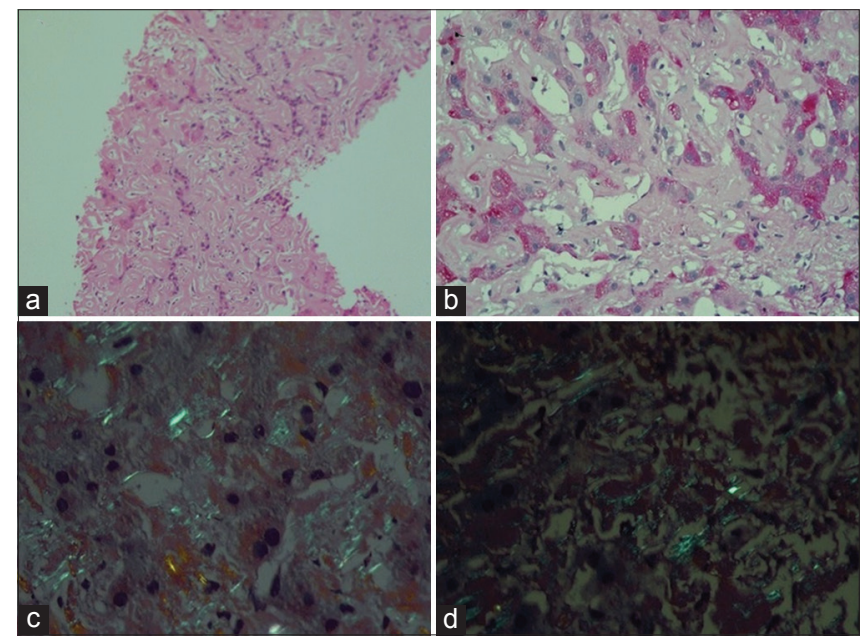

Figure 1: Showing the liver biopsy specimen and various stains. (a) Liver tissue showing amorphous eosnophilic material in the sinusoids (H\&E x100); (b) Faint PAS positive material in the sinusoids (x200); (c) Apple green birefringence on polarizing microscopy of congo red stain $(x 400)$; (d) Congophilia persists after treatment witn $\mathrm{KMnO} 4$ (x400)

negative for hepatocellular inclusions. PERL stain negative for iron, Negative for fibrosis on masons trichrome stain.

USG abdomen- Hepatomegaly with coarse parenchymal echoes suggestive of CLD, splenomegaly and moderate ascites s/o CLD with PHTN. GB sludge no evidence of biliary obstruction/cholecystitis. Prostate enlarged with median lobe hypertrophy and calcified. Portal hepatic venous flow visible but poor flow.

CECT- hepatomegaly with thrombosed hepatic veins. Rt middle and left wavy hepatic regions. Widened interlobar fissures, collaterals, mild splenomegaly, moderate ascites with features Acute Budd chairi syndrome.

Normal renal function. Urine dark yellow, protein $-3+$, RBC's- 8-10 ph-5.5, Glucose -nil, ketones -trace, Pus cells 2-4, fine granular cast present cal oxalate crystal were present. Urine PCR-1.8 g. Serum electrophoresis did not show M-band. Serum immunofixation showed Kappa, Gamma globulin and IgG positivity, negative for myeloma and IgA and IgM not detected. Kappa free light chain $-568.0 \mathrm{mg} / \mathrm{dl}$ (3.3-19.4), lambda free light chain $72 \mathrm{mg} / \mathrm{dl}(5.7-26.3)$. Infectious virology- negative. 2D-echocardiography was normal, Abdominal fat biopsy was done which confirmed amyloidosis. Prostate, kidney biopsy was not done as we lost the patient.

\section{DISCUSSION}

Here we report a case of primary hepatic amyloidosis that presented with features of hepatic vein obstruction. The association of Budd-Chiari's syndrome with amyloidosis 
may be related to the increased risk of thrombosis observed in the latter disease. Our patient had hepatomegaly with constitutional symptoms, features of hepatic failure together with raised alkaline phosphatase and GGT, Subnephrotic range proteinuria and micro hematuria, with features of prostatomegaly and Budd Chairi syndrome. Renal biopsy owing to his poor general condition and prostate biopsy was not done. Radiological findings of hepatic involvement are non-specific. Pre-contrast and contrast enhanced CECT abdomen reveals enlarged liver with three small focal lesions seen in segment VIII, V and VII measuring $4 \mathrm{~mm}, 9 \times 11 \mathrm{~mm}$ and $7 \mathrm{~mm}$ respectively. These lesions are not visualized in unenhanced scans and shows progressive enhancement in arterial, portal phase with intense enhancement in the venous phase. Lesions homogenizing in delayed scan. Heterogeneous enhancement of hepatic parenchyma in delayed phase. All hepatic veins are thrombosed with hypodense lumen more evident in the venous phase. Anti-SAA Immunoperoxidase staining from liver biopsy was also negative. Hereditary forms of amyloidosis including lysosome form were considered. There was no evidence of spontaneous rupture, cardiac, or nervous system involvement, tuberculosis, rheumatoid arthritis, SLE, crohns disease, or evidence of other common inflammatory diseases. However, renal involvement could not be established conclusively with renal biopsy.

Though amyloidosis is considered as a systemic disease, 10\%-20\% cases can be localized. ${ }^{7}$ To our knowledge primarily isolated hepatic involvement of liver in amyloidosis has rarely been described in the literature. Though it is possible that the patient has yet exhibited the evidence of systemic disease, hepatic involvement can occur in both primary and secondary types of amyloidosis (AL/AA).

In primary amyloidosis fibrillar protein which is deposited is a fragment of the variable immunoglobulin either commonly by light (and/or rarely heavy) chain. Whereas in secondary type the protein is the amino acid terminus of the acute phase protein SAA. ${ }^{7}$ Secondary amyloidosis with hepatic involvement can be seen in chronic inflammatory disorders and infections including multiple myeloma, tuberculosis, rheumatoid arthritis, familial Mediterranean fever, Crohn's disease, Reiter's syndrome, ankylosing spondylitis, Sjögren's syndrome, dermatomyositis, vasculitis, chronic osteomyelitis, bronchiectasis, cystic fibrosis, systemic lupus erythematosus (SLE). ${ }^{7}$

In hepatic amyloidosis, extensive compression of hepatocytes by the amyloid deposits results in atrophy of hepatocyte. This occurs due to amyloid deposits in the liver parenchyma along the sinusoids \& within the spaces of disse or within the blood vessel walls. Massive infiltration amyloid results in hepatomegaly with rubber elastic consistency. This results in "lardaceous liver" appearance on cut- surface. ${ }^{8}$

The clinical spectrum of HA includes involuntary weight loss, Hepatomegaly was found in 79 patients $(81 \%)$. Eightytwo patients (89\%) had proteinuria, and 81 patients $(86 \%)$ had elevated serum alkaline phosphatase levels. Seventy-six patients $(83 \%)$ had either a serum or urine monoclonal protein. Hepatic ruputure and bleeding post liver biopsy was rare. Clinical suspicion of amyloidosis is also very challenging. The median survival rate in these patients is 8.5 months.'

A study using antibodies against three different regions of immunoglobulin lambda light chain for the Immunohistochemical analysis of liver biopsy samples from the cases of immunoglobulin lambda light chain amyloidosis showed that the amyloid deposits may not be homogeneous in the liver and that molecular heterogeneity of amyloid fibril protein or a difference in the mode of deposition results in the histopathological heterogeneity of AL amyloid deposits. ${ }^{10}$ Immunohistochemistry using anti-kappa and anti lambda antibodies are useful in Immunohistochemical classification and diagnosis of AL type amyloidosis. However it has its own limitations owing to cross reactivity between anti-kappa and anti-lambda antibodies. ${ }^{10}$

There have been case reports of hepatic involvement in amyloidosis including one where the presentation was a liver SOL, in the setting of plasma cell dyscrasias, but isolated hepatic involvement is a rare entity, ${ }^{2}$ with nephrotic syndrome, ${ }^{3}$ intra hepatic cholestasis, ${ }^{3,4,11,12}$ Budd chairi syndrome. ${ }^{5}$

Here we present a rare entity of isolated hepatic amyloidosis, with hepatic failure, Subnephrotic proteinuria and Budd chairi syndrome and gamma globulin, lambda and kappa positive. He was advised to undergo liver transplantation, he could not be initiated on chemotherapeutic agents, as he deteriorated and passed away. This case is a learning curve in the field of medicine, as diagnosis is challenging, and the treatment options available are also limited.

"Written informed consent was obtained from the patient's kin for publication of this Case report and any accompanying images. A copy of the written consent is available for review by the Editor-in-Chief of this journal."

\section{REFERENCES}

1. Gullu BE, Hatem I, Ozbay G, Tuzuner N and Sonsuz A. A rare case of primary systemic amyloidosis presenting with hepatic failure. Turkish J Gastroenterol 2011;22(1):113-114. 
2. Son RC, Chang JC and Choi JH. Primary hepatic amyloidosis: Report of an unusual case presenting as a mass. Korean $\mathrm{J}$ Radiol 2016;12(3):382-385.

3. Georgiades CS, Neyman EG, Barish $M$ and Fishman EK. Amyloidosis: Review and CT manifestations. Radiographics 2004;24(2):405-416.

4. Gavilan JC, Bermudez FJ, Marquez A, Sanchez-Carrillo JJ and Gonzalez-Santos P. Hepatic amyloidosis as cause of severe intrahepatic cholestasis. An Med Interna 2003;20(1):25-27.

5. Tsuji H, Murai K, Kobayashi K, Nishimura J, Sumiyoshi K, Akagi $\mathrm{K}$, et al. Multiple myeloma associated with Budd-Chiari syndrome. Hepatogastroenterology 1990;2(37):97-99.

6. Paliard $P$, Bretagnolle $M$, Collet $P$, Vannieuwenhyse $A$ and Berger $\mathrm{F}$. Inferior vena cava thrombosis responsible for chronic Budd-Chiari syndrome during hepatic and digestive amyloidosis. Gastroenterol Clin Biol 1983;7(11):919-922.

7. Urban BA, Fishman EK, Goldman SM, Scott WW, Jones B, Humphrey RL, et al. CT evaluation of amyloidosis: Spectrum of disease. Radiographics 1993;13(6):1295-308.

8. Monzawa S, Tsukamoto T, Omata K, Hosoda K, Araki T and Sugimura K. A case with primary amyloidosis of the liver and spleen: Radiologic findings. Eur J Radiol 2002;41(3):237-241.

9. Park MA, Mueller PS, Kyle RA, Larson DR, Plevak MF and Gertz MA. Primary (AL) hepatic amyloidosis: Clinical features and natural history in 98 patients. Medicine 2003;82(5):291-298.

10. Kiyama M, Hoshii $\mathrm{Y}$, Cui D, Kawano H, Kanda T and Ishihara T. Immunohistochemical and immunochemical study of amyloid in liver affected by systemic Alambda amyloidosis with antibodies against three different regions of immunoglobulin lambda light chain. Pathol Int 2007;57(6):343-350.

11. Goenka MK, Bhasin DK, Vasisth RK and Dhawan S. Hepatic amyloidosis presenting with severe intrahepatic cholestasis. J Clin Gastroenterol 1996; 23(2):134-136.

12. Bandyopadhyay SK, Bandyopadhyay R and Dutta A. Primary amyloidosis presenting as intrahepatic cholestasis. Indian $\mathrm{J}$ Pathol Microbiol 2006; 49(4):557-559. 